\title{
Frailty and quality of life in adult survivors of childhood cancer
}

\author{
Kirsten K. Ness, PT, PhD, Carrie R. Howell, PhD, and Kari L. Bjornard, MD, MPH
}

\begin{abstract}
Introduction-Adult survivors of childhood cancer are at risk for frailty, a state of diminished physiologic reserve, typically seen in older adults, associated with morbidity and mortality. They are also at risk for poor quality of life. This review explores potential associations between frailty and quality of life and proposes potential interventions that may prevent or remediate frail health.
\end{abstract}

Areas covered and literature search-A literature search using MEDLINE and EMBASE was undertaken using key words: childhood cancer survivors and each of the following: frailty, physical performance, emotional health, and quality of life. 796 abstracts were reviewed for relevance. Reference lists for retrieved articles were consulted for pertinent manuscripts ( $N=40)$. Recent literature (from January 2013 to November 2016) was obtained using the key words: aging and frailty and quality of life. The final reference list includes 44 documents.

Expert opinion-Childhood cancer survivors are at risk for frailty and less than optimal quality of life. Poor emotional health may contribute to this association. Further research is needed to better explain these associations and to provide a foundation for the development of interventions designed to prevent and remediate frail health and suboptimal quality of life in this population.

\section{Keywords}

Frailty; childhood cancer survivors; fitness; physical performance; emotional health; quality of life; exercise

\section{Introduction}

The proportion of children diagnosed with cancer who survive five years after their original cancer diagnosis has improved from 66\% in 1975 to over 83\% in 2008 [1]. The most recent Cancer Statistics Review indicates that there were over 408,000 survivors of childhood cancer living in the United States in 2013 [1]. Nearly 290,000 of these individuals were younger than age 40 , with years of potential to actively and significantly contribute to their family life, communities, and to society [1,2]. Unfortunately for survivors, treatment for childhood cancer, while curative, does not come without risk for late effects. Surgical intervention, therapeutic radiation, and chemotherapy administration do not always spare normal tissue, in some cases leaving the young survivor with less than optimal organ system structure and function $[3,4,5]$. While most young adult cancer survivors do not have clinically significant chronic disease, some experience symptoms that interfere with or limit

Corresponding: Kirsten K. Ness, PT, PhD, Member, Epidemiology and Cancer Control, St. Jude Children's Research Hospital, 262 Danny Thomas Place, Memphis, TN 38105, 901-595-5157 (p), kiri.ness@ stjude.org.

Financial and competing interests' disclosure: The authors report no conflicts of interest. 
their abilities to participate fully in life roles at home, at work, in the community, or during recreational activities. These symptoms may include fatigue [6,7], shortness of breath [8], or an inability to fully engage in activities that require exertion [9]. Such symptoms are all markers of reduced physiologic capacity, and may be associated with reduced quality of life.

Recent data indicate that adult survivors of childhood cancer are at increased risk for frailty, a phenotype characterized by diminished physiologic reserve, and most commonly described in populations of older adults. In older adults and in childhood cancer survivors, frailty, represented by a constellation of three or more of: low lean muscle mass, muscle weakness, slow walking speed, low energy expenditure, and/or self-reported exhaustion, is associated with new onset of chronic disease and mortality. Additionally, in older adults, and in persons with chronic disease, frailty is associated with diminished quality of life $[10,11,12,13]$, an association that is heightened among persons who also have depression [14]. The association between frailty and quality of life, and the additional potential contribution of depression to this association have not been investigated among adult survivors of childhood cancer. However, there are data suggesting that physical performance limitations, less than optimal fitness, and poor emotional health influence perceived well-being among adult survivors of childhood cancer. This is important because individual components of the frailty phenotype and problems with emotional health are amenable to rehabilitation efforts in older adults [15, $16,17]$ and in those with chronic disease $[14,18]$. Thus, among adult survivors of childhood cancer who are frail and/or who are experiencing depression, providing intervention has potential to prevent new onset chronic disease, extend lifespan, and improve overall quality of life. This review provides an overview of frailty and fitness outcomes among the growing population of childhood cancer survivors, of the literature documenting the associations between physical performance/fitness and quality of life among childhood cancer survivors, of the known contributions of poor emotional health to this association, and also suggests that there may be potential interventions to improve physical performance/fitness and to optimize quality of life in childhood cancer survivors.

\section{Frailty and fitness among adult survivors of childhood cancer}

Recent evidence supports the hypothesis that adult survivors of childhood cancer have diminished physiologic reserve, and that frailty among childhood cancer survivors occurs decades earlier than expected. In one investigation, physical performance was characterized among survivors of childhood brain tumors and compared them to age-gender-race, and zip code-matched members of the general population [19]. Results of this study indicated that among 78 survivors of childhood brain tumors (median age 22 (range 18-58) years), cardiopulmonary fitness and muscle strength values (in the unaffected extremity in cases of hemiplegia) were not only lower than the comparison group, but also very close to fitness levels and strength values observed among persons in their seventh decade of life (Figure 1) [19]. In another investigation, neuromuscular impairments in survivors of childhood acute lymphoblastic leukemia (ALL) were characterized. Among 415 survivors of childhood ALL ten or more years from diagnosis, with a median age of 35 (range 21-52) years, $46.5 \%$ had six minute walk distances, and $30.1 \%$ had knee extension strength values more than 1.3 standard deviations less than expected when compared to age and sex matched normative data. The average distances walked in six minutes among these ALL survivors were similar 
to predicted distances for individuals aged 50-60 years (Figure 2) [20]. Furthermore, data presented at the 2011 American Society for Clinical Oncology annual meeting demonstrated that muscle weakness and poor endurance were prevalent among adult survivors of Hodgkin lymphoma (HL) who were at least 10 years from their original diagnosis. Those with measured performance in the lowest $10^{\text {th }}$ percentile of age- and sex-predicted norms for strength and endurance were classified as having severe limitations. Among 196 eligible HL survivors, 188 (median age 40 (range 26-61) years; median time since diagnosis 26 (range 11-45) years) participated. Among these HL survivors, $22.3 \%$ had severe ankle weakness, $21.8 \%$ had severe quadriceps weakness, and $31.9 \%$ performed in the severely impaired range on the six minute walk test [21].

These results extend to a large cohort of adult survivors of childhood cancer. In a preliminary investigation designed to examine the frailty phenotype, the Fried Frailty Model was applied to 1,922 members of the St. Jude Lifetime (SJLIFE) Cohort Study. Among these $10+$ year survivors of childhood cancer (50.3\% male, median [range] age 32 [18-59] years, age at diagnosis 6 [0-21] years), physical function was evaluated and "pre-frailty" and "frailty" defined as 2 and $\geq 3$ of: sarcopenia, decreased muscle strength, poor endurance, slow walking speed, and low levels of activity. Nearly one-third (30.3\%) met pre-frailty criteria; $7.9 \%$ met frailty criteria. Frailty increased with age $(6.1 \%, 11.1 \%, 21.7 \%$ for $18-29$, $30-39$ and $40-59$ years respectively), was more common among females (16.2\% vs. $2.7 \%$ males), and highest among leukemia (8.2\%), CNS tumor (7.4\%), non-Hodgkin lymphoma $(10.0 \%)$, and bone tumor $(7.4 \%)$ survivors. Those exposed to chest $(8.2 \%$ vs. $5.0 \%)$ or brain radiation $(7.4 \%$ vs. $3.5 \%)$, anthracyclines $(5.9 \%$ vs. $4.7 \%)$, or alkylating agents $(6.6 \%$ vs. $3.8 \%$ ) were more likely to be frail than those who were not exposed. Percentages of prefrailty and frailty in this young adult cohort of survivors were slightly higher than those in the Cardiovascular Health Study (30.3\% vs. $22.2 \%$ and $7.9 \%$ vs. $7.2 \%$ ), which included older adults (65-101 years) and among whom this phenotype was first described [22]. These preliminary findings that young adult survivors of childhood cancer experience frailty at rates similar to those observed in persons who are several decades older are compelling, important and warrant further investigation.

\section{Associations between fitness and quality of life in childhood cancer survivors}

While there are well documented associations between the contributions of specific sociodemographic factors, cancer diagnoses, presence of chronic disease, and poor quality of life in physical domains among survivors of childhood cancer [23, 24, 25, 26, 27, 28, 29], knowledge of how the specific frailty phenotype impacts quality of life among young adult survivors of childhood cancer has not been documented. Nevertheless, there is evidence that physical function/fitness, both perceived and measured with objective performance testing, is associated with participation and quality of life in this population. In a report from the Childhood Cancer Survivor Study (CCSS), associations between limitations in physical performance and scoring $\leq 40$ on the summary and subscales of the SF-36 among 7,147 adult survivors of childhood cancer were documented [30]. Associations between limitations in physical performance and suboptimal quality of life were highest for the physical 
component summary, and the physical function, role physical, bodily pain, and general health subscales. Nagarajan et al [31], also using data from the CCSS, documented an association between self-reported disability status (from the Tumor Extremity Salvage Score (TESS)) and score on the Quality of Life-for Cancer Survivors Instrument (QOL-CS). Among 528 long-term survivors of childhood extremity tumors, 21 years from diagnosis, those who reported themselves as disabled scored lower on the QOL-CS (6.0 vs. 7.2, p < $0.001)$ than those who did not report themselves as disabled. Similarly, in a study from the SJLIFE cohort, Fernandez-Pineda et al [32] defined poor fitness among 206 extremity sarcoma survivors (median age 36 years) as walking a two or more standard deviations shorter distance in six minutes than an age- sex-matched comparison group. These authors documented a 4.83 (95\% CI 1.95-11.99) fold increased risk of scoring $\leq 40$ on the physical component summary of the SF-36 in the 81 survivors with poor fitness compared to the 125 survivors without poor fitness.

\section{Contributions of emotional distress to the association between physical performance/fitness and quality of life}

Poor emotional health, the onset of which appears to begin early in survivorship, is an independent predictor of problems with physical quality of life among childhood cancer survivors. However, the role of emotional health as a prior predictor, mediator, or moderator of the association between physical performance/fitness and quality of life is not well understood. Li et al [33] evaluated physical, psychological, and social well-being among 137 children and adolescents (aged 9-16 years) who were between one and five year survivors of childhood cancer. Depressive symptoms were present in $52.6 \%$ of children and mean selfesteem scores were lower for cancer survivors than for a sample of their peers. Semistructured interviews with a subset of children indicated that problems with fatigue and school performance were contributing factors to both depression and poor self-esteem. Blaauwbroek et al [34], in an analysis of chronic health conditions and quality of life among 313 childhood cancer survivors reported that, even after accounting for organ system specific chronic disease, problems in the psychosocial domain were associated with nearly a standard deviation $(-8.8,95 \% \mathrm{CI}-13.4$ to -4.1$)$ decrease in score on the physical function subscale of the SF-36. Additionally, Huang et al [35], in an analysis of data from the SJLIFE cohort $(\mathrm{N}=1,667)$, reported that symptoms, both somatic and those representing emotional health, explain 56\% of the variance in physical component summary of the SF-36 even after accounting for sociodemographic and treatment factors. In the large CCSS study $(\mathrm{N}=7,147)$ mentioned above, among adult survivors of childhood cancer, the independent association between emotional health and quality of life was also evaluated. As expected, emotional health was strongly associated with scoring $\leq 40$ on the mental component summary and the bodily pain, general health, social function, role-emotional, and mental health subscales of the SF-36. However, after accounting for sociodemographic factors, physical performance and executive function, emotional health was also an independent predictor of scoring $\leq 40$ on the physical component summary, and the physical function, role physical, and vitality subscales of the SF-36. In fact, the impact of poor emotional health was stronger than the impact of physical performance on the role-physical (OR 4.38 (95\% CI 3.66-5.23) vs. 2.99 (95\% CI 2.59-3.54)) and vitality (OR 9.54 (7.57-12.02) vs. 1.72 (1.50-1.97) subscales. In 
these models, physical performance, executive function, and emotional health were not collinear, nor did they significantly interact [30].

The analyses, summarized above, were all based on cross sectional data. However, in a longitudinal evaluation of CCSS data, Nolan et al [36], reported associations between both disfigurement and walking with a limp, in addition to obesity as predictors of future problems with quality of life among adult survivors of adolescent onset cancers $(\mathrm{N}=2,064)$. Although this analysis did not specifically evaluate emotional health, another analysis identified an association between disfigurement and problems with quality of life in this cohort [37], indicating that initial difficulty adjusting to cancer related hair loss, scarring from surgery or radiation, or loss of a limb because of a bone or soft tissue tumor may impact long-term quality of life. Further work carefully documenting physical performance, emotional health, and quality of life starting with younger cohorts of childhood cancer survivors, and following them longitudinally to determine temporal associations will be necessary to determine the need for and timing of interventions designed to improve quality of life.

\section{Potential interventions for fitness and quality of life in childhood cancer survivors}

Even though physical exercise interventions in frail older adults appear to improve physical performance/fitness outcomes and quality of life, we were unable to identify any randomized interventions that have addressed these issues in adult survivors of childhood cancer. Some initial work has focused on targeting children during or soon after cancer therapy in order to prevent functional loss. A systematic review supports the initial efficacy of physical exercise interventions during or soon after treatment for childhood cancer to improve physical performance and fitness, but only if adherence to the intervention is optimized [38]. Given the documented impact of emotional health on quality of life, combining physical exercise interventions with psychosocial training during this time period would seem reasonable if the goal of intervention was to also improve quality of life. However a small randomized trial evaluating combined physical exercise and psychosocial training indicates that intervention does not result in appreciable differences in either fitness or health related quality of life if administered during or in the first year after cancer therapy [39]. It may be that interventions delivered this early that also require significant exertion and cognitive attention are too difficult for children to participate in while they are acutely ill.

It will be important to optimize timing of interventions for this population. It is possible that starting a preventive intervention after initial recovery from the cancer therapy may be easier for survivors and their families. Castellano et al [29] reported an association between the practice of weekly physical exercise and concurrent quality of life among 42 adolescent survivors of childhood cancer (12-19 years of age) at least one year off curative therapy. To augment success, it will also be important for interventions to include components that promote self-confidence and accommodate any physical performance limitations that survivors are experiencing. For instance, Boman et al [40], in a study of 528 adult survivors 
of childhood central nervous system (CNS) tumors, indicated that both perceived global health and functional abilities are important indicators of self-confidence for participation in sport/physical activity. Also, an exercise intervention delivered at home among twelve children (aged 5-10 years) with acute lymphoblastic leukemia, with a remote exercise coach who contacted them weekly during the maintenance phase of chemotherapy, had excellent adherence (82\% of prescribed session) and demonstrated improved fitness outcomes [41]. This intervention was tailored to each child's current abilities and modified accordingly to allow the child to be successful throughout the course of the intervention and to improve self-confidence. Unfortunately, quality of life and emotional health were not evaluated as part of this study.

Interventions to address the needs of survivors who are already frail and/or who have diminished quality of life are also needed. Bray et al (Table 1) [14] recently proposed a literature based, structured exercise prescription for pre-frail and frail older adults that could be tested for efficacy among adult survivors of childhood cancer. This type of intervention is safe and has demonstrated preliminary efficacy to improve fitness in small samples of childhood sarcoma and ALL survivors. Smith et al [42] reported no adverse events during the intervention, as well as $86 \%$ adherence to a 12 week home based aerobic and strengthening program among five adult survivors of childhood cancer who had been exposed to anthracyclines and who had subclinical cardiomyopathy with cardiac ejection fractions between 40 and 55\%. Jarvela et al [43] reported improved peak oxygen uptake and core and lower body strength among seventeen 16 to 30 year old survivors of childhood ALL after 16 weeks of participation in a structured home based strengthening program. Adult survivors of childhood cancer with frailty and poor health related quality of life are likely to be amenable to such an intervention, as survivors of childhood cancer with chronic conditions who also report poor quality of life are more likely than those who do not report poor quality of life to seek and utilize medical, physical therapy, and/or chiropractic services to address their performance/fitness problems. Michel et al [44], in a study of 141 adult (1845 years) survivors of childhood cancer five or more years from diagnosis, reported an association between lower score on the physical component summary of the SF-36 and the desire for continued specialist care in long-term follow-up. Montgomery et al [45], in a study of 9,289 adult survivors of childhood cancer, reported that $12.4 \%$ utilized chiropractic and $9.2 \%$ utilized physical therapy services in the two years preceding completion of a questionnaire. Those with a musculoskeletal (OR 1.8, 95\% CI 1.1-2.9), neurological (OR $3.4,95 \%$ CI 1.6-6.9), or cardiovascular (OR 3.3, 95\% CI 1.6-6.9) chronic condition who utilized PT/chiropractic services were more likely to report poor physical health related quality of life than those with the conditions who did not utilize services.

\section{Summary}

The number of childhood cancer survivors living into adulthood is increasing. A subset of them are at risk for both frail health (limitations in physical performance/poor fitness) and less than optimal quality of life and problems with emotional health may contribute to this association. However, there is a need for further examination of the associations between frailty, emotional health, and quality of life in this population. Information from observational, cross sectional, but particularly longitudinal studies, is needed to further 
characterize this relationship, as well as provide a foundation for the development of intervention studies. The timing of such studies will be important, as children on or immediately after cancer therapy may have difficulty participating when they are acutely ill. Prevention early in survivorship and remediation in long-term survivors appears promising and is amenable to study.

\section{Expert commentary}

This review summarizes the literature describing the risk for frailty, and less than optimal quality of life, among childhood cancer survivors. It also discusses the potential impact of emotional health on this association. The literature is not complete. Thus the need for future study examining the associations between frail health and quality of life in survivors of childhood cancer along the survivorship continuum is discussed. In addition, this review identifies interventions, used in other populations, with potential to impact quality of life by remediating or preventing frail health.

\section{Five-year view}

A study looking at the progression of frailty and its association with quality of life in the St. Jude Lifetime Cohort among childhood cancer survivors eighteen years of age or older, and at least 10 years from their original cancer diagnosis is ongoing (NCT02256137).

Participants had clinical and functional assessments at a baseline visit; second visits five years after the baseline visit are underway. Both visits include measures of emotional health and quality of life. These data should provide robust information further documenting the potential association between frail health and quality of life in adult survivors of childhood cancer, and allow researchers to identify potential targets for intervention.

In the interim, a preliminary study (NCT02501460) designed to investigate the impact of resistance training and supplementation on a component of frailty, lean muscle mass, may provide important information on the ability of adult survivors of childhood cancer to respond to an intervention proven in other populations.

Finally, biological mechanisms of frailty (accelerated aging) are also undergoing investigation, with one of the National Cancer Institute's Pediatric Provocative Questions [46] designed to attract research in this area: "PPQ-9: What are the underlying molecular mechanisms that cause accelerated aging seen in some pediatric cancer survivors?" Its intent is to stimulate research to identify both treatment and genetic risk factors associated with onset of early aging, and to identify mechanisms underlying the aging phenotype. Data from research funded by this mechanism should provide early targets to prevent frail health and promote optimal quality of life.

\section{Acknowledgments}

This work was supported by the National Cancer Institute under Grant CA21765; and the American Lebanese and Syrian Associated Charities. 


\section{References}

1. Howlader, N., Noone, AM., Krapcho, M., et al. SEER Cancer Statistics Review, 1975-2013. Bethesda, MD: 2016. [October 26, 2016]. Available from: http://seer.cancer.gov/csr/1975_2013/, based on November 2015 SEER data submission, posted to the SEER web site, April 2016

2. Mariotto AB, Rowland JH, Ries LA, et al. Multiple cancer prevalence: a growing challenge in longterm survivorship. Cancer epidemiology, biomarkers \& prevention: a publication of the American Association for Cancer Research, cosponsored by the American Society of Preventive Oncology. 2007; 16:566-71.

3. Bowers DC, Adhikari S, El-Khashab YM, et al. Survey of long-term follow-up programs in the United States for survivors of childhood brain tumors. Pediatr Blood Cancer. 2009; 53:1295-301. [PubMed: 19688835]

4. Hudson MM, Mulrooney DA, Bowers DC, et al. High-risk populations identified in Childhood Cancer Survivor Study investigations: implications for risk-based surveillance. J Clin Oncol. 2009; 27:2405-14. [PubMed: 19289611]

5. Oeffinger KC, Mertens AC, Sklar CA, et al. Chronic health conditions in adult survivors of childhood cancer. The New England journal of medicine. 2006; 355:1572-82. [PubMed: 17035650]

6. Johannsdottir IM, Hjermstad MJ, Moum T, et al. Increased prevalence of chronic fatigue among survivors of childhood cancers: A population-based study. Pediatr Blood Cancer. 2012; 58:415-20. [PubMed: 21425447]

7. Mulrooney DA, Ness KK, Neglia JP, et al. Fatigue and sleep disturbance in adult survivors of childhood cancer: a report from the childhood cancer survivor study (CCSS). Sleep. 2008; 31:27181. [PubMed: 18274275]

8. Mertens AC, Yasui Y, Liu Y, et al. Pulmonary complications in survivors of childhood and adolescent cancer. A report from the Childhood Cancer Survivor Study. Cancer. 2002; 95:2431-41. [PubMed: 12436452]

9. Ness KK, Leisenring WM, Huang S, et al. Predictors of inactive lifestyle among adult survivors of childhood cancer: a report from the Childhood Cancer Survivor Study. Cancer. 2009; 115:1984-94. [PubMed: 19224548]

10. Verlaan S, Aspray TJ, Bauer JM, et al. utritional status, body composition, and quality of life in community-dwelling sarcopenic and non-sarcopenic older adults: A case-control study. Clin Nutr. Nov 27.2016 Epub.

11. Kojima G, Iliffe S, Morris RW, et al. Frailty predicts trajectories of quality of life over time among British community-dwelling older people. Qual Life Res. 2016; 25:1743-50. [PubMed: 26747318]

12. Lee IC, Chiu YH, Lee CY. Exploration of the importance of geriatric frailty on health-related quality of life. Psychogeriatrics. 2016; 16:368-75. [PubMed: 26756915]

13. Provencher V, Sirois MJ, Emond M, et al. Frail older adults with minor fractures show lower health-related quality of life (SF-12) scores up to six months following emergency department discharge. Health Qual Life Outcomes. 2016; 14:40. [PubMed: 26956158]

14. Bray NW, Smart RR, Jakobi JM, et al. Exercise prescription to reverse frailty. Appl Physiol Nutr Metab. 2016; 41:1112-6. [PubMed: 27649859]

15. de Labra C, Guimaraes-Pinheiro C, Maseda A, et al. Effects of physical exercise interventions in frail older adults: a systematic review of randomized controlled trials. BMC Geriatr. 2015; 15:154. [PubMed: 26626157]

16. Tarazona-Santabalbina FJ, Gomez-Cabrera MC, Perez-Ros P, et al. A Multicomponent Exercise Intervention that Reverses Frailty and Improves Cognition, Emotion, and Social Networking in the Community-Dwelling Frail Elderly: A Randomized Clinical Trial. J Am Med Dir Assoc. 2016; 17:426-33. [PubMed: 26947059]

17. Chang SF, Wen GM. Association of frail index and quality of life among community-dwelling older adults. J Clin Nurs. 2016; 25:2305-16. [PubMed: 27161863]

18. Lisiak M, Uchmanowicz I, Wontor R. Frailty and quality of life in elderly patients with acute coronary syndrome. Clin Interv Aging. 2016; 11:553-62. [PubMed: 27217737]

19. Ness KK, Morris EB, Nolan VG, et al. Physical performance limitations among adult survivors of childhood brain tumors. Cancer. 2010; 116:3034-44. [PubMed: 20564409] 
20. Ness KK, Hudson MM, Pui CH, et al. Neuromuscular impairments in adult survivors of childhood acute lymphoblastic leukemia: associations with physical performance and chemotherapy doses. Cancer. 2012; 118:828-38. [PubMed: 21766297]

21. Marina, N., Hudson, M., Mulrooney, D., et al. Comparison of health status between upper and lower extremity sarcoma survivors: a report from the childhoood cancer survivor studyOctober 2830. Aukland, New Zealand: 2011.

22. Fried LP, Tangen CM, Walston J, et al. Frailty in older adults: evidence for a phenotype. The journals of gerontology Series A, Biological sciences and medical sciences. 2001; 56:M146-56.

23. Rueegg CS, Gianinazzi ME, Rischewski J, et al. Health-related quality of life in survivors of childhood cancer: the role of chronic health problems. J Cancer Surviv. 2013; 7:511-22. [PubMed: 23784593]

24. Kanellopoulos A, Hamre HM, Dahl AA, et al. Factors associated with poor quality of life in survivors of childhood acute lymphoblastic leukemia and lymphoma. Pediatr Blood Cancer. 2013; 60:849-55. [PubMed: 23335116]

25. Schultz KA, Chen L, Chen Z, et al. Health conditions and quality of life in survivors of childhood acute myeloid leukemia comparing post remission chemotherapy to BMT: a report from the children's oncology group. Pediatr Blood Cancer. 2014; 61:729-36. [PubMed: 24285698]

26. Yi J, Kim MA, Tian T. Perceived long-term and physical health problems after cancer: adolescent and young adult survivors of childhood cancer in Korea. Eur J Oncol Nurs. 2014; 18:145-50. [PubMed: 24361038]

27. Alessi D, Dama E, Barr R, et al. Health-related quality of life of long-term childhood cancer survivors: a population-based study from the Childhood Cancer Registry of Piedmont, Italy. Eur J Cancer. 2007; 43:2545-52. [PubMed: 17869094]

28. Bradley Eilertsen ME, Jozefiak T, Rannestad T, et al. Quality of life in children and adolescents surviving cancer. Eur J Oncol Nurs. 2012; 16:185-93. [PubMed: 21945190]

29. Girard P, Auquier P, Barlogis V, et al. Symptomatic osteonecrosis in childhood leukemia survivors: prevalence, risk factors and impact on quality of life in adulthood. Haematologica. 2013; 98:108997. [PubMed: 23645686]

30. Ness KK, Gurney JG, Zeltzer LK, et al. The impact of limitations in physical, executive, and emotional function on health-related quality of life among adult survivors of childhood cancer: a report from the Childhood Cancer Survivor Study. Archives of physical medicine and rehabilitation. 2008; 89:128-36. [PubMed: 18164342]

31. Nagarajan R, Clohisy DR, Neglia JP, et al. Function and quality-of-life of survivors of pelvic and lower extremity osteosarcoma and Ewing's sarcoma: the Childhood Cancer Survivor Study. Br J Cancer. 2004; 91:1858-65. [PubMed: 15534610]

32. Fernandez-Pineda I, Hudson MM, Pappo AS, et al. Long-term functional outcomes and quality of life in adult survivors of childhood extremity sarcomas: a report from the St. Jude Lifetime Cohort Study. J Cancer Surviv. Jun 4.2016 Epub.

33. Li HC, Lopez V, Joyce Chung OK, et al. The impact of cancer on the physical, psychological and social well-being of childhood cancer survivors. Eur J Oncol Nurs. 2013; 17:214-9. [PubMed: 22898653]

34. Blaauwbroek R, Stant AD, Groenier KH, et al. Health-related quality of life and adverse late effects in adult (very) long-term childhood cancer survivors. Eur J Cancer. 2007; 43:122-30. [PubMed: 16987653]

35. Huang IC, Brinkman TM, Kenzik K, et al. Association between the prevalence of symptoms and health-related quality of life in adult survivors of childhood cancer: a report from the St Jude Lifetime Cohort study. J Clin Oncol. 2013; 31:4242-51. [PubMed: 24127449]

36. Nolan VG, Krull KR, Gurney JG, et al. Predictors of future health-related quality of life in survivors of adolescent cancer. Pediatr Blood Cancer. 2014; 61:1891-4. [PubMed: 24664999]

37. Kinahan KE, Sharp LK, Seidel K, et al. Scarring, disfigurement, and quality of life in long-term survivors of childhood cancer: a report from the Childhood Cancer Survivor study. J Clin Oncol. 2012; 30:2466-74. [PubMed: 22614987] 
38. Braam KI, van der Torre P, Takken T, et al. Physical exercise training interventions for children and young adults during and after treatment for childhood cancer. Cochrane Database Syst Rev. 2016; 3:CD008796. [PubMed: 27030386]

39. van Dijk-Lokkart EM, Braam KI, van Dulmen-den Broeder E, et al. Effects of a combined physical and psychosocial intervention program for childhood cancer patients on quality of life and psychosocial functioning: results of the QLIM randomized clinical trial. Psychooncology. 2016; 25:815-22. [PubMed: 26509236]

40. Boman KK, Hornquist L, De Graaff L, et al. Disability, body image and sports/physical activity in adult survivors of childhood CNS tumors: population-based outcomes from a cohort study. $\mathrm{J}$ Neurooncol. 2013; 112:99-106. [PubMed: 23354653]

41. Esbenshade AJ, Friedman DL, Smith WA, et al. Feasibility and initial effectiveness of home exercise during maintenance therapy for childhood acute lymphoblastic leukemia. Pediatric physical therapy: the official publication of the Section on Pediatrics of the American Physical Therapy Association. 2014; 26:301-7.

42. Smith WA, Ness KK, Joshi V, et al. Exercise training in childhood cancer survivors with subclinical cardiomyopathy who were treated with anthracyclines. Pediatric blood \& cancer. 2013

43. Jarvela LS, Kemppainen J, Niinikoski H, et al. Effects of a home-based exercise program on metabolic risk factors and fitness in long-term survivors of childhood acute lymphoblastic leukemia. Pediatr Blood Cancer. 2012; 59:155-60. [PubMed: 22184098]

44. Michel G, Gianinazzi ME, Eiser C, et al. Preferences for long-term follow-up care in childhood cancer survivors. Eur J Cancer Care (Engl). 2016; 25:1024-33. [PubMed: 27550385]

45. Montgomery M, Huang S, Cox CL, et al. Physical therapy and chiropractic use among childhood cancer survivors with chronic disease: impact on health-related quality of life. Journal of cancer survivorship: research and practice. 2011; 5:73-81. [PubMed: 20922492]

46. National Cancer Institute. Research Answers to NCI's Pediatric Provocative Questions (R01). 2016. Available from: https://grants.nih.gov/grants/guide/pa-files/PAR-16-218.html 


\section{Key Issues}

- Evidence suggests that adult survivors of childhood cancer are at risk for frail health, emotional distress, and less than optimal quality of life, the association between frailty and quality of life.

- $\quad$ Because individual components of the frailty phenotype and problems with emotional health are amenable to rehabilitation efforts in older adults, and in those with chronic disease, providing interventions for adult survivors of childhood cancer has potential to prevent new onset chronic disease, extend lifespan, and improve overall quality of life in this population.

- $\quad$ There is a need for research to better explain the association between frail health and quality of life in childhood cancer survivors.

- There is also a need for research among childhood cancer survivors evaluating the effects of interventions known to remediate frail health and improve quality of life in other populations. Childhood cancer survivors have unique needs; interventions that prevent or remediate frail health in other populations may need modifications to maximize efficacy. 


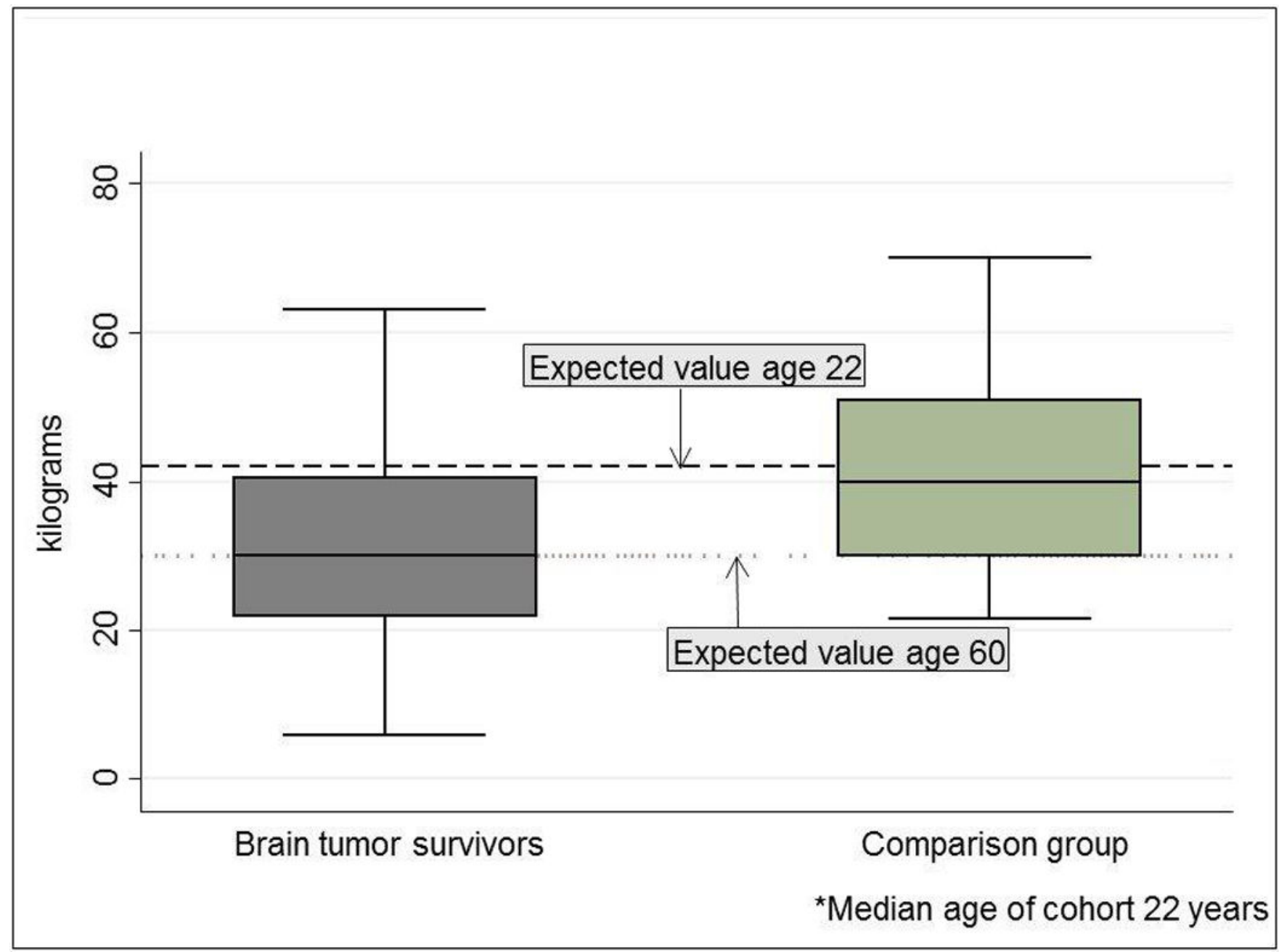

Figure 1.

Handgrip strength among brain tumor survivors and an age and sex matched comparison group 


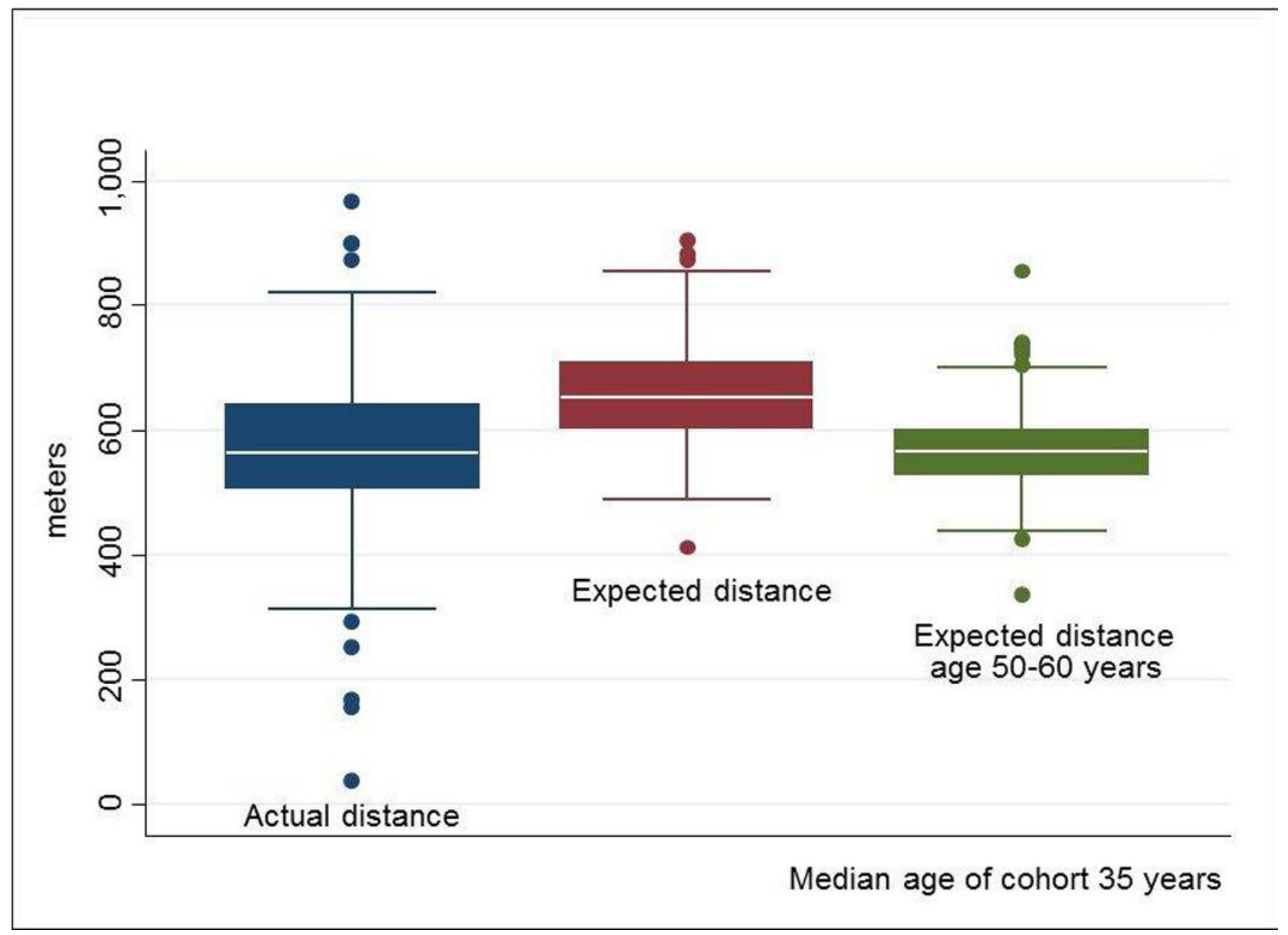

Figure 2.

Six minute walk distance among acute lymphoblastic leukemia survivors 


\section{Table 1}

Proposed exercise intervention [14] for adults with frailty

\begin{tabular}{|l|l|l|l|l|}
\hline & Resistance training & Aerobic training & Flexibility & Balance \\
\hline $\begin{array}{l}\text { Pre-frail } \\
\text { 60 minutes } \\
\text { per session) }\end{array}$ & $\begin{array}{l}\text { 2-3 times per week at 80\% of } \\
\text { 1-Repetition Maximum } \\
20 \text { minutes }\end{array}$ & $\begin{array}{l}\text { 2-3 times per week at perceived } \\
\text { rate of exertion 3-4 on a 10 point } \\
\text { scale (somewhat hard) } \\
10 \text { minutes }\end{array}$ & $\begin{array}{l}\text { 2-3 times per week at } \\
\text { perceived rate of exertion } \\
3-4 \text { on a 10 point scale } \\
\text { (somewhat hard) } \\
10 \text { minutes }\end{array}$ & $\begin{array}{l}\text { 2-3 times per week at } \\
\text { p-4 on a 10 point scale } \\
\text { (somewhat hard) } \\
20 \text { minutes }\end{array}$ \\
\hline $\begin{array}{l}\text { Frail } \\
\text { (45 minutes } \\
\text { per session) }\end{array}$ & $\begin{array}{l}\text { 2-3 times per week at 80\% of } \\
10 \text { minutes }\end{array}$ & $\begin{array}{l}\text { 2-3 times per week at perceived } \\
\text { rate of exertion 3-4 on a 10 point } \\
\text { scale (somewhat hard) } \\
20 \text { minutes }\end{array}$ & $\begin{array}{l}\text { 2-3 times per week at } \\
\text { perceived rate of exertion } \\
\text { 3-4 on a 10 point scale } \\
\text { (somewhat hard) } \\
7 \text { minutes }\end{array}$ & $\begin{array}{l}\text { perceived rate of exertion } \\
\text { 3-4 on a 10 point scale } \\
\text { (somewhat hard) } \\
8 \text { minutes }\end{array}$ \\
\hline
\end{tabular}

